\title{
How to Trade Off between Regularization and Image Similarity in Non-rigid Registration?
}

\author{
Pascal Cachier \\ Projet Epidaure, INRIA, Sophia-Antipolis, France \\ Pascal.Cachier@inria.fr
}

\section{Introduction}

In this work, we propose a new subdivision of intensity-based non-rigid registration algorithms composed of two classes:

Standard Intensity Based (SIB) algorithms. In these algorithms, the same transformation is used to compute the intensity similarity measure while being constrained to remain smooth.

Pair-and-Smooth (P\&S) algorithms. These algorithms proceed in two steps, which may alternate or not. In the first step, they look for corresponding points, using the intensity similarity measure. In the second step, they approximate these pairings using a smooth non-rigid transformation. Block-matching, optical flow based and ICP based algorithms belong to this class.

The underlying fundamental difference with SIB registration is that the smoothness of the estimated transformation is balanced with a geometric measure (i.e. a distance with the corresponding features found during the first step) instead of an intensity measure. This has a strong impact on the smoothness of the registration, which we study in the case of competitive registration.

\section{Comparison of Competitive SIB and P\&S Algorithms}

Competitive algorithms, as defined in [1], use an additional energy $E_{\text {reg }}$ (e.g. the linear elastic energy) to regularize the estimation problem on top of the intensity similarity energy $E_{\text {sim }}$. In the case of competitive SIB algorithms, the registration is done by minimizing the weighted sum $E(I, J, T)=E_{\text {sim }}(I, J, T)+$ $\lambda E_{\text {reg }}(T)$ : a trade-off is made between two quantities with different physical dimensions, a geometric measure $\left(E_{\text {reg }}\right)$ and an intensity measure $\left(E_{\text {sim }}\right)$. The intensity similarity is related to the amount of change in the intensity necessary to go from one image to the other, which is not uniformly proportional to the amount of motion necessary to deform one image to the other. Consequently, smoothness largely depends on the local variation of the similarity measure, and so on the local contrast of the image.

On the other hand, competitive P\&S algorithms alternate between finding corresponding features (generally points) using the intensity similarity, and smoothing these pairings. The trade-off here is done during the second step, between geometric quantities : this explains why in practice the smoothness of the solutions found by $\mathrm{P} \& \mathrm{~S}$ algorithms are much more uniform and controllable. 


\subsection{Experiment}

We have registered a synthetically deformed image with Asym and PASHA algorithms. Asym is a SIB algorithm that minimizes the following classical energy using a gradient descent: $E(I, J, T, \lambda)=\int(I-J \circ T)^{2}+\lambda \int\|d T\|^{2}$. PASHA is a $\mathrm{P} \& \mathrm{~S}$ algorithm that minimizes the following hybrid energy, introduced in [1] for P\&S registration: $E(I, J, C, T)=\int(I-J \circ T)^{2}+\sigma \int\|C-T\|^{2}+\sigma \lambda \int\|d T\|^{2}$. We have run the experiment with a large range of parameters, and kept the result minimizing the average error for both algorithms (fig. 1).
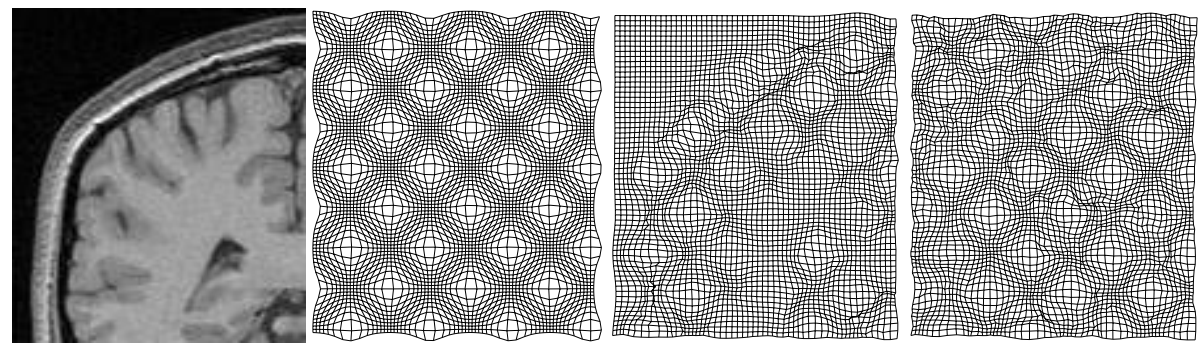

Fig. 1. Left to right: the test image, the synthetic deformation applied to it, and the best result achievable with Asym and PASHA.

With Asym the smoothness of the transformation is non-uniform because of the inhomogeneity of the trade-off: the transformation is almost not estimated on plain areas of the image, while still irregular on the edges in the image. Its average distance to the original transformation is only 1.27 pixel. With PASHA, the quality of the estimation is very uniform, which enables a $35 \%$ smaller average distance of 0.83 pixel.

\subsection{Two Priors for Non-rigid Registration}

PASHA has two parameters $\sigma$ and $\lambda$, corresponding to two separate priors: the level of noise in the image and the smoothness of the transformation. In the classical approach there is only one regularization parameter, which theoretically accounts only for an image noise prior. Unfortunately, in practice it is hardly so: for example, in the previous experiment, best results are obtained for moderate regularization strengths while the images are virtually noiseless. Using two priors enable to separate image reliability and transformation smoothness. We show in [1] that this enables quantitatively better results, whatever the level of noise in the images or the smoothness of the transformation.

\section{References}

1. P. Cachier and N. Ayache. Regularization in Image Non-Rigid Registration: I. Trade-Off between Smoothness and Similarity. Technical Report RR-4188, INRIA, 2001. http://www.inria.fr/rrrt/index.en.html. 